\title{
Unicameral Bone Cyst
}

\section{A Retrospective Study of Three Surgical Treatments}

\author{
Anthony D. Sung BS, Megan E. Anderson MD, \\ David Zurakowski PhD, Francis J. Hornicek MD, PhD, \\ Mark C. Gebhardt MD
}

Received: 15 October 2007/ Accepted: 7 July 2008/Published online: 5 August 2008

(C) The Association of Bone and Joint Surgeons 2008

\begin{abstract}
Between 1979 and 2004, 167 patients younger than 20 years were treated surgically for humeral or femoral unicameral bone cysts with either injection of corticosteroids (steroids), curettage plus bone grafting (curettage), or a combination injection of steroids, demineralized bone matrix, and bone marrow aspirate (SDB) at Children's Hospital of Boston and Massachusetts General Hospital (mean followup, 7.3 years; range, 1 month-27 years). Outcomes included treatment failure (defined clinically as subsequent pathologic fracture or need for retreatment to prevent pathologic fracture) and complications. Information
\end{abstract}

Each author certifies that he or she has no commercial associations (eg, consultancies, stock ownership, equity interest, patent/licensing arrangements, etc) that might pose a conflict of interest in connection with the submitted article.

One of the authors (ADS) has received funding from the Pasteur Summer Research Program and the Harvard Medical School Office of Enrichment Programs for support of this study.

Each author certifies that his or her institution has approved the human protocol for this investigation, that all investigations were conducted in conformity with ethical principles of research, and that informed consent for participation in the study was obtained.

\section{A. D. Sung}

Harvard Medial School, Boston, MA, USA

M. E. Anderson ( $)$

330 Brookline Ave, Boston, MA 02215, USA

e-mail: manders6@bidmc.harvard.edu

M. E. Anderson, D. Zurakowski, M. C. Gebhardt

Children's Hospital Boston, Boston, MA, USA

M. E. Anderson, M. C. Gebhardt

Beth Israel Deaconess Medical Center, Boston, MA, USA

F. J. Hornicek

Massachusetts General Hospital, Boston, MA, USA was obtained from medical records and by telephone questionnaire. After one treatment, $84 \%$ of cysts treated with steroids experienced failed treatment versus $64 \%$ with curettage and 50\% with SDB. For unicameral bone cysts requiring retreatment (regardless of first treatment), $76 \%$ retreated with steroids had failed treatment versus $63 \%$ with curettage and $71 \%$ with SDB. Curettage was associated with the lowest rate of posttreatment pathologic fractures and highest rate of pain and other complications. Multivariate logistic regression indicated treatment with steroids alone and younger age were independent predictors of failure. We believe SDB is a reasonable first treatment for unicameral bone cysts in the humerus and femur in patients younger than 20 years, being less invasive yet comparable to curettage in preventing recurrence.

Level of Evidence: Level IV, prognostic study. See the Guidelines for Authors for a complete description of levels of evidence.

\section{Introduction}

Unicameral bone cysts (UBCs) are benign, single-chambered, fluid-filled lesions that occur most often in the proximal humerus or femur of skeletally immature individuals [41]. These cysts weaken the cortex, predisposing the bone to pathologic fracture (Fig. 1). Although quiescent UBCs often spontaneously resolve as patients approach skeletal maturity [41], this happens as little as 7\% to $15 \%$ of the time in symptomatic UBCs that cause pain or pathologic fracture, and treatment usually is needed to prevent additional fractures [1, 14, 28].

Although UBCs were first recognized by Virchow in 1876 [40], their etiology remains unknown, hindering 


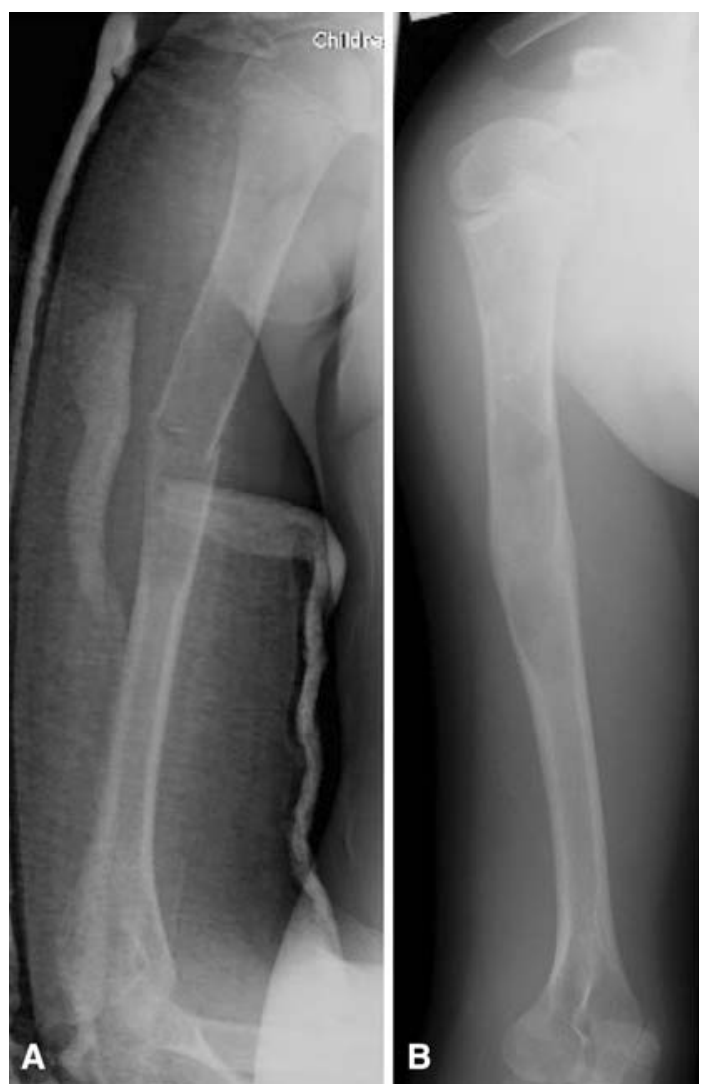

Fig. 1A-B (A) A radiograph shows a UBC with pathologic fracture. (B) Although the fracture has healed, the cyst persists.

development of a definitive treatment that targets their pathogenesis. Some theorize, based on electron microscopy, they are intraosseous synovial cysts [26], whereas others propose they are the result of trauma [18]. Analysis of cyst fluid reveals elevated levels of prostaglandins [35], suggesting a possible role in the pathogenesis and supporting treatment with steroid injection [34], whereas the theory that UBCs form in response to venous congestion of the intramedullary space [8] has prompted others to use trephination, high-speed saline injection, and other methods that restore circulation $[3,6,15,22]$.

In part because we do not know the etiology of UBCs, there is no consensus for the best treatment [41]. Controversy exists regarding when treatment should occur or if treatment is necessary [24], because most cysts resolve spontaneously near skeletal maturity. The main indication for surgical intervention is preventing pathologic fracture. However, uncertainty exists regarding the effectiveness of various treatments: even for curettage and steroids, two of the more traditional and well-studied methods, wide ranges of failure rates after initial treatment are reported: 22\% [28] to $47 \%$ [13] after curettage and $41 \%$ [10] to $78 \%$ [42] after steroids. Numerous other treatments have been suggested, ranging from subtotal resection $[12,25]$, to flexible intramedullary nailing $[9,11,31,33]$, to placement of Kirschner wires [6, 7]. In addition to steroids [4, 34], percutaneous injection has been attempted with demineralized bone matrix (DBM) [21], bone marrow [23, 42], or a combination of DBM and bone marrow [19, 32]. No previous study has examined injection with a combination of steroids, DBM, and bone marrow aspirate (SDB).

We proposed that SDB, combining the antiinflammatory effects of steroids and the osteoinductive properties of DBM and bone marrow with a minimally invasive approach, would be a more effective treatment for UBCs than steroids alone. Our rationale was based on previous studies that showed good healing with these components individually [23, 34, 41]. We hypothesized that, by combining them, they would act synergistically.

Our study compared SDB with two traditional treatments: percutaneous steroid injection (steroids) and open curettage plus bone grafting (curettage) [13, 28]. As a result of the potential for recurrence, repeat treatments often are needed to achieve healing, sometimes as many as five or six [41]. Because the ideal treatment would cure the patient after only one attempt, our primary outcome was the failure rate after the initial procedure. Secondary outcomes included failure after a second procedure (when required), incidence of subsequent pathologic fractures, and complications such as pain and deformity. We also sought to identify other factors besides treatment that might be independent predictors of treatment failure. We hypothesized SDB would result in the lowest failure rate after one procedure, the lowest failure rate after a second procedure, and the fewest complications compared with the traditional treatments of steroid injection and curettage.

\section{Materials and Methods}

We performed a computerized search of operative reports at Children's Hospital Boston and Massachusetts General Hospital to identify all patients younger than 20 years who were surgically treated for UBCs in the humerus or femur between 1979 and 2004. We found 167 patients who received one of the three study treatments and had sufficient followup to determine whether they had a treatment failure or survived more than 2 years without failure. Twenty-one surgeons participated in the treatment of these patients, but the majority was performed by the senior author (MCG) and the technical aspects of placing the needles in the cyst or curettage were similar for all surgeons. Information was obtained from medical records. We also attempted to contact as many patients as possible to obtain further followup, administering a telephone questionnaire to $99(59 \%)$ patients (parents if the patient was a minor) to determine if treatment failures or complications 
occurred. The questions were similar to those asked during a typical outpatient followup visit. Although we also reviewed radiographs, radiographic data were not collected because outcomes ultimately were assessed clinically. We defined failure as refracture or the physician's recommendation to retreat to prevent refracture. Although this end point is inherently subjective, previous studies have noted reliance on the objective of radiographic recurrence resulted in unnecessary treatment of recurrences that were small and clinically insignificant (ie, did not weaken the cortex or predispose to fracture) [27].

Diagnosis was made on radiographic and clinical criteria and, in most cases, confirmed by pathologic examination. The finding of a radiolucent metaphyseal lesion on plain film with a characteristic lytic appearance with thinning of the cortex, especially in the context of pathologic fracture or pain, was considered diagnostic and sufficient for inclusion in the study. MRI usually was not needed. In cysts treated by injection, the color of the fluid obtained at the initial entry of the cyst was serous or clear without blood. On aspirating the fluid, it usually became bloody, and filling the cyst with contrast supported the diagnosis. In the majority of cases, the cyst fluid was sent for cytologic evaluation to exclude other neoplasms. In cysts that were curetted, a specimen was sent for pathologic examination.

There is no consensus or official guidelines for when to treat UBCs. In our study, indications for surgery were a history of pathologic fracture or the surgeon's assessment of risk of impending fracture as assessed by clinical history of pain and radiographic findings of UBC and cortical thinning. Occasionally, the size of the cyst and thickness of the cyst wall influenced the decision to operate, but no exact biomechanical formula was used. Decisions were made based on clinical opinion, and procedure selection was at the choice of the surgeon and patient or parents. Similarly, there were no formal rules for when retreatment was necessary; the decision was based on the physician's clinical opinion, taking into account factors such as wall thickness and extent of cyst resolution. In general, femoral lesions were more likely to be curetted. When the diagnosis was in doubt, surgeons were more likely to do an open biopsy to secure a tissue diagnosis. Age had no effect on treatment decision. Only seven patients had internal fixation, and all of those were for femoral lesions that had curettage. The number of patients who wore a cast was not recorded.

Of the 167 patients enrolled, $56 \%$ received steroids as their initial treatment, $23 \%$ had curettage, and $20 \%$ had SDB (Table 1). The average age of the patients was 9.5 years; $72 \%$ were male; $69 \%$ had lesions in the humerus and $31 \%$ had lesions in the femur. Seventy-five percent presented with pathologic fractures; $72 \%$ were treated at Children's Hospital of Boston and 28\% were treated at Massachusetts General Hospital. Patients treated with curettage were more likely to have a UBC in the femur and less likely to have had a previous pathologic fracture $(\mathrm{p}<0.001)$. Patients treated with SDB had less followup on average than those treated with steroids or curettage. This is partly because SDB was a newer technique first used in the mid1990 s. Overall demographics were similar to those reported in other large studies for age, gender, location, and history of pathologic fracture [41]. We had an average of 7.3 years (range, 1 month-27 years) followup per person.

Curettage was performed by dissecting down to the bone and creating a cortical window sufficient to view the entire cavity. After the fluid was removed, the fibrous membrane lining the cyst wall was curetted. A power burr was not used routinely to extend the curettage. The cyst then was packed with corticocancellous allograft bone chips. The allograft bone was acquired from an American Association of Tissue Banks-accredited tissue bank.

Percutaneous steroid injection was performed by inserting two needles into the cyst. Injection of radiopaque contrast and fluoroscopy were used to confirm contact with the entire cyst. The cyst fluid was aspirated, the cyst was irrigated with saline, and methylprednisolone (40-120 mg, depending on the size of the cyst, age of the patient, and surgeon preference) was injected. The two-needle technique allowed circulation and efflux of excess fluid through

Table 1. Patient demographics

\begin{tabular}{|c|c|c|c|c|c|}
\hline Demographic & Steroids $(\mathrm{n}=94)$ & Curettage $(\mathrm{n}=39)$ & $\operatorname{SDB}(\mathrm{n}=34)$ & $\mathrm{p}$ Value & Overall $(\mathrm{n}=167)$ \\
\hline Average age (years) & 9.09 & 10.27 & 9.74 & $0.21 *$ & 9.50 \\
\hline Male & $71(76)$ & $31(80)$ & $26(77)$ & $0.89^{\dagger}$ & $128(77)$ \\
\hline Located in humerus & $81(86)$ & $5(13)$ & $29(85)$ & $<0.001^{\dagger}$ & 115 (69) \\
\hline Initial fracture & $82(87)$ & $19(49)$ & $25(74)$ & $<0.001^{\dagger}$ & $126(75)$ \\
\hline Treated at $\mathrm{CHB}$ & $69(73)$ & $24(62)$ & $28(82)$ & $0.25^{\dagger}$ & $121(72)$ \\
\hline Average followup (years) & 7.77 & 8.92 & 4.03 & $<0.001^{*}$ & 7.28 \\
\hline
\end{tabular}

Values in parentheses represent percentage of treatment group; *p values based on analysis of variance; ${ }^{\dagger} \mathrm{p}$ values based on Pearson's chi square test; SDB = combination of steroids, demineralized bone matrix, and bone marrow aspirate; CHB = Children's Hospital of Boston. 
the outflow needle. Data were not available for the amount of saline used. SDB injection was similar to steroid injection, except bone marrow aspirated from the patient's iliac crest through 11- to 15-gauge bone marrow harvest needles was mixed with the DBM and methylprednisolone before the combination was injected into the cyst. In general, 13- to 15-gauge bone marrow harvest needles were used for injection of steroids or SDB. No other adjuvants were used. In all instances, the DBM used was Grafton ${ }^{\circledR}$ gel (Musculoskeletal Transplant Foundation, Edison, NJ).

Outcomes included failure after the first treatment, failure after the second treatment (when needed), further pathologic fractures despite treatment, and persistence of pain and other complications. Pain, numbness, or tingling that persisted at least 1 year after surgery was scored as a binary variable (present or absent). When recording data for complications, we took into account all aspects of the procedure (eg, morbidity from bone marrow aspiration for SDB) rather than simply complications related to the cyst. We collected data for age, gender, location, history of pathologic fracture, and hospital, to test how these variables influenced failure. Information on size of the cyst, wall thickness, and percentage of cyst resolution was unavailable for many cases and not collected. Univariate analysis was performed to determine the association between failure and age, gender, location, history of pathologic fracture, hospital, and treatment using the chi square test. Logistic regression analysis was applied to derive the odds of recurrence (OR) for each treatment with 95\% confidence intervals (CIs) [17]. Survivorship was determined using the Kaplan-Meier method with treatments compared by the log rank test [2]. Error bars for survivorship curves represent $95 \%$ CIs as calculated by Greenwood's formula [16]. To adjust for possible confounders among the six variables, stepwise logistic regression was used and probability of recurrence for each treatment was determined by maximum likelihood estimation [20]. In designing this retrospective study, to ensure $80 \%$ statistical power, a minimum sample size of 20 patients for each variable being examined was required (treatment and other covariates) [30]. Analysis was conducted using SPSS ${ }^{\circledR}$ (Version 15.0; SPSS Inc, Chicago, IL). Two-tailed values of $\mathrm{p}<0.05$ were considered significant.

\section{Results}

The failure rate after initial treatment was $84 \%$ (79 of 94) with steroids, $64 \%$ (25 of 39 ) with curettage, and $50 \%$ (17 of 34) with SDB $(\mathrm{p}<0.001)$ (Table 2). Logistic regression showed steroids were associated with a higher risk of failure than curettage (OR, 3.0; 95\% CI, 1.3-6.9) or SDB (OR, 5.3; 95\% CI, 2.2-12.6). There was no difference between curettage and SDB $(\mathrm{p}=0.23)$. Subgroup analysis showed higher failure rates with steroids regardless of location or age; this difference was statistically significant for lesions in the humerus $(\mathrm{p}<0.001)$ and in patients between 10 and 14 years old $(\mathrm{p}=0.016)$. This was supported by logistic regression analysis; taking into account age, gender, location, history of pathologic fracture, and hospital, steroids remained an independent predictor of failure compared with curettage (OR, 2.5; 95\% CI, 1.1-6.2) or SDB (OR, 5.2; 95\% CI, 2.1-12.8). This also was supported by Kaplan-Meier analysis, which additionally showed steroids were associated with earlier failures than curettage or SDB (Fig. 2).

Of the 121 patients who had the first treatment fail, $97 \%$ (117) received a second treatment using one of the three study methods; of these, $85 \%$ had sufficient followup to determine the failure rate after retreatment was $76 \%$ (51 of 67) with steroids, $63 \%$ (five of eight) with curettage, and $71 \%$ (17 of 24 ) with SDB. There were no significant differences between treatments $(\mathrm{p}=0.66)$. These results were

Table 2. Failures after first treatment

\begin{tabular}{|c|c|c|c|c|c|}
\hline Failures & Steroids $(\mathrm{n}=94)$ & Curettage $(\mathrm{n}=39)$ & $\mathrm{SDB}(\mathrm{n}=34)$ & p Value* & Overall $(n=167)$ \\
\hline Overall & 79/94 (84) & 25/39 (64) & $17 / 34(50)$ & $<0.001$ & $121 / 167(73)$ \\
\hline \multicolumn{6}{|l|}{ By location } \\
\hline Femur & $12 / 13(92)$ & $23 / 34(68)$ & 4/5 (80) & 0.21 & $39 / 52(75)$ \\
\hline Humerus & 67/81 (83) & $2 / 5(40)$ & $13 / 29(45)$ & $<0.001$ & 83/115 (72) \\
\hline \multicolumn{6}{|l|}{ By age } \\
\hline $0-4$ years & $7 / 8(88)$ & $5 / 6(83)$ & 2/3 (67) & 0.72 & 14/17 (82) \\
\hline 5-9 years & 43/49 (88) & 8/11 (72) & $9 / 14(64)$ & 0.11 & $60 / 79(81)$ \\
\hline $10-14$ years & $26 / 32(81)$ & $11 / 19(58)$ & $6 / 15(40)$ & 0.016 & 43/66 (65) \\
\hline $15-19$ years & $3 / 5(60)$ & $1 / 3(33)$ & $0 / 2(0)$ & 0.53 & 4/10 (40) \\
\hline
\end{tabular}

Values are expressed as the number of failures/the total number of patients in a group; values in parentheses represent percentage of failures; *p values based on Pearson's chi square test; SDB = combination of steroids, demineralized bone matrix, and bone marrow aspirate. 


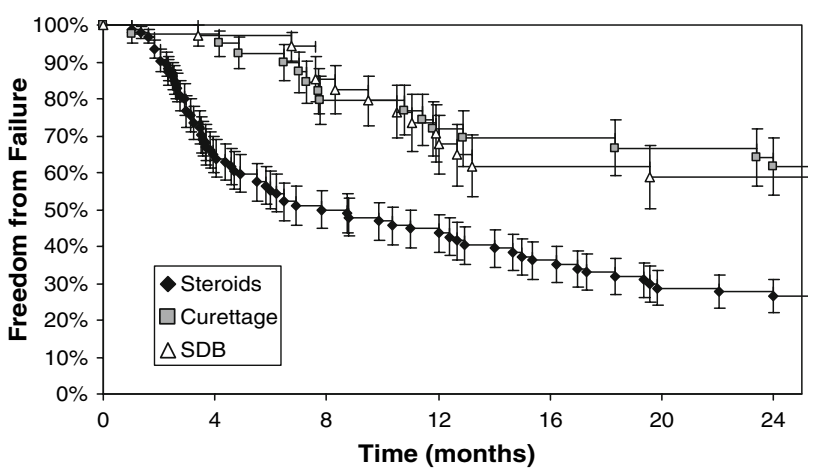

Fig. 2 Kaplan-Meier analysis shows patients initially treated by steroids have earlier and more failures.

analyzed independent of first treatment because there were not enough data to stratify by first and second treatments.

Eighteen percent (17 of 94) of patients initially treated with steroids had a subsequent pathologic fracture despite treatment versus $2.6 \%$ (one of 39 ) with curettage and $12 \%$ (four of 34) with SDB $(\mathrm{p}=0.053)$. Logistic regression showed steroids were associated with a higher risk of refracture than curettage (OR, 8.4; 95\% CI, 1.1-65.4); there were no differences between SDB and other treatments $(\mathrm{p}>0.10)$.

The results were reversed for patient reports of occasional pain, numbness, or tingling: 5.3\% (five of 94) of patients who received steroids as their first treatment experienced these complications versus $21 \%$ (eight of 39) with curettage and $8.8 \%$ (three of 34) with SDB $(\mathrm{p}=0.025)$. Logistic regression showed curettage was associated with a higher risk of pain than steroids (OR, 4.6, 95\% CI, 1.4-15.1); there were no differences between SDB and other treatments $(\mathrm{p}>0.10)$.

There were no differences between groups when looking at other complications such as deformity or growth disturbance; $5.3 \%$ (five of 94) of patients who received steroids as their first treatment reported other complications versus $7.7 \%$ (three of 39 ) with curettage and $2.9 \%$ (one of $34)$ with SDB $(\mathrm{p}=0.67)$. There was no extra morbidity from iliac marrow aspiration. Although connection with the venous system, as seen by contrast injections into UBCs, presents the risk of embolization of injected particulate matter (ie, DBM, bone marrow), we did not find any clinically significant occurrences of this event.

Univariate predictors of failure after one treatment include younger age $(\mathrm{p}<0.001)$ and treatment with steroids $(\mathrm{p}<0.001)$ (Table 3$)$. Gender $(\mathrm{p}=0.76)$, hospital $(\mathrm{p}=0.90)$, initial fracture $(\mathrm{p}=0.49)$, and location $(\mathrm{p}=0.62)$ were not predictive of failure. Multiple logistic regression confirmed younger age $(\mathrm{p}=0.004)$ and treatment with steroids $(\mathrm{p}<0.001)$ were independent predictors of failure. We constructed a model showing the relationship among age, treatment, and failure rate (Fig. 3).
Table 3. Univariate predictors of failure after one treatment

\begin{tabular}{lc}
\hline Predictor & p Value* \\
\hline Gender & 0.76 \\
Age & $<0.001$ \\
Hospital & 0.90 \\
Initial fracture & 0.49 \\
Location & 0.62 \\
Treatment & $<0.001$ \\
\hline
\end{tabular}

* p values based on Pearson's chi square test.

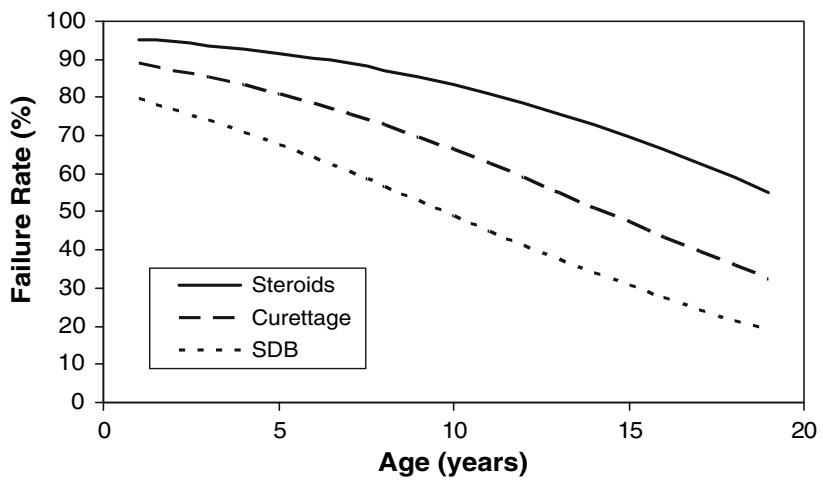

Fig. 3 Using multiple logistic regression, we constructed an algorithm to predict the failure rate after one treatment as a function of treatment and patient's age. At every age, patients treated by steroids have higher failure rates than those treated by curettage or SDB.

Table 4. Failure rates in the femur versus humerus by treatment

\begin{tabular}{lrrrrr}
\hline Treatment & Femur & Failed & Humerus & Failed & $\mathrm{p}$ Value* \\
\hline First & & & & & \\
$\quad$ Steroids & 13 & $12(92)$ & 81 & $67(83)$ & 0.69 \\
$\quad$ Curettage & 34 & $23(68)$ & 5 & $2(40)$ & 0.33 \\
$\quad$ SDB & 5 & $4(80)$ & 29 & $13(45)$ & 0.34 \\
$\quad$ Total treated & 52 & $39(75)$ & 115 & $82(71)$ & 0.71 \\
Second & & & & & \\
$\quad$ No treatment & 1 & & 3 & & \\
$\quad$ Steroids & 20 & $16(80)$ & 57 & $35(61)$ & 0.28 \\
$\quad$ Curettage & 6 & $3(50)$ & 4 & $2(50)$ & 0.93 \\
$\quad$ SDB & 12 & $9(75)$ & 18 & $8(44)$ & 0.23 \\
$\quad$ Total treated & 38 & $28(74)$ & 79 & $45(57)$ & 0.40 \\
\hline
\end{tabular}

Values in parentheses represent percentage of treatment group; *p values based on Fisher's exact test comparisons between femur and humerus groups; SDB = combination of steroids, demineralized bone matrix, and bone marrow aspirate.

Although this study was conducted over 25 years, there were no significant differences when comparing lesions treated early versus late in the study. In addition, there were no significant differences when comparing lesions in the femur versus humerus (Table 4; overall, $\mathrm{p}=0.71$; steroids, $\mathrm{p}=0.69$; curettage, $\mathrm{p}=0.33$; $\mathrm{SDB}, \mathrm{p}=0.34$ ) or 
when comparing lesions in the femur that were curetted and internally fixed versus lesions that simply were curetted $(p=0.12)$.

\section{Discussion}

The choices facing the physician when presented with a child with a UBC are deciding whether to observe or treat and, if the decision is made to treat, how to treat. The main indication for treatment is prevention of pathologic fracture. Reliable methods to determine fracture risk only recently have been published [37]. That issue is not the goal of this article; our question is, once the decision is made to treat, how to treat. Although the eventual success of UBC treatment almost always is assured, multiple attempts often are required. We sought to identify which of the three treatment methods we were using during the time of this study has the lowest failure rate per attempt and a minimum of complications based on retrospective analysis. We also sought to identify other predictors of failure.

Our study has certain limitations, including inherent selection bias like all retrospective studies. Indications and treatment selection were based on clinical criteria and physician discretion rather than objective criteria. Multiple surgeons were involved, and although this may not be ideal, it is reflective of how patients are treated in practice. Most of the procedures were performed by the senior author (MCG) who was consistent in the use of the techniques throughout the study period. Another concern is, although multiple logistic regression showed treatment with steroids was an independent predictor of a higher rate of initial treatment failure, we were only able to take into account the potential confounders of age, femoral versus humeral location, gender, hospital, and history of pathologic fracture; data on size, activity, and proximal versus distal location of the cyst were unavailable. Although we did not see a significant difference between lesions in the femur versus humerus or lesions that were curetted and internally fixed versus those that were just curetted, our study was not designed and powered to detect such differences. We did see a significant difference in the length of followup: SDB is a newer treatment and patients were followed an average of 4.03 years versus 7.77 and 8.92 years for steroids and curettage, respectively; shorter followup may cause late adverse events to be missed. Another consideration is, when evaluating outcomes of a second treatment, our results may have been biased by the first treatment received, because there were not enough patients to stratify by first and second treatments. Similarly, our findings concerning rates of refracture, pain, and other complications may have been influenced by subsequent treatments that patients may have received or the effects of pathologic fractures. Finally, although the unique radiographic and clinical appearances of UBCs are considered diagnostic and often were confirmed by aspiration/cytology or biopsy, pathologic specimens were not available for every case.

Nonetheless, our study of 167 patients is one of the largest in the literature, with patients having an average followup of 7.3 years, and we directly compared different treatments. Our finding that steroids were associated with an $84 \%$ failure rate after one treatment is comparable to the $76 \%$ failure rate after one procedure in a series of 163 patients [34]; other smaller series have reported failure rates of $41 \%$ [10], 47\% [13], 50\% [29], and 78\% [42] after one injection. Although others have reported using doses as much as $240 \mathrm{mg}$ [34], we are not aware of any doseresponse curve that suggests higher doses are more effective. Our finding that curettage was associated with a $64 \%$ failure rate after one treatment is higher than the $47 \%$ reported by one large study [13], and others have reported lower failure rates of $38 \%$ [29], 36\% [38], 25\% [39], and even $22 \%$ [28]. One possible explanation for the differences in failure rates in our study versus published rates is the longer followup in our study, which may have resulted in detection of more late failures.

Our other findings with regard to complications and factors influencing outcome are supported by the literature. Curettage and other invasive procedures have long been associated with high complication rates [29], although we could not find any studies that reported on pain or discomfort. The relationship between younger age and higher failure rates has been noted in other studies $[14,27,29,38$, 39]. Although most consider age 10 as the cutoff between patients who do well and those who fare poorly, we believe there is a more linear relationship between younger age and higher failure rates. We also examined whether outcomes differed between males and females because some studies reported higher rates of failure in males [27, 39] and others reported higher rates of failure in females [27]. These results may have been confounded by factors such as age and treatment. According to our multivariate regression analysis, taking into account age, treatment, location, and hospital, gender had no effect on outcome.

In addition to SDB, other new methods have been proposed for treating UBCs, many with excellent results. Trephination and drilling with or without Kirschner wire placement have resulted in failure rates after one treatment of $27 \%$ [22], 30\% [6], and 52\% [36]. Even greater successes have been reported with intramedullary nailing, with failure rates after one treatment of $0 \%[11,33]$ and $6 \%$ [31]. However, intramedullary nailing remains controversial because local relapse of UBC is usually tolerable and rarely limb-threatening, and one has to balance the invasiveness and complications of a procedure with disease 
control. Furthermore, one study noted $28 \%$ of patients needed another operation because the nail became too short for the growing bone [31]. For other injection techniques, an $18 \%$ failure rate has been reported after one DBM injection [21], and failure rates of $11 \%$ [19] and 22\% [32] have been reported after one injection of DBM plus bone marrow. However, early promising results may later be shown as false; although a $0 \%$ failure rate initially was reported after one injection of bone marrow [23], a later study reported a 50\% failure rate [42], and a study comparing bone marrow injection with steroids reported failure rates of $57 \%$ versus $49 \%$, showing no advantage with bone marrow [5]. This same observation applies to our finding of a failure rate of $50 \%$ after one SDB injection.

Because many factors may impact outcome, it is difficult to compare results across studies; study populations may differ with regard to age, location of cyst, and size. In addition, the uncertainty surrounding the pathogenesis of UBCs makes it difficult to anticipate effects of treatments. The action of SDB may be mediated by the osteoinductive properties of DBM and bone marrow coupled with the inhibitory effect of steroids on phospholipase and prostaglandin formation (and subsequent decrease in osteoclastic activity).

Until the pathogenesis of UBC is elucidated, the primary focus of patient care is empiric treatment. These findings show SDB is more effective as an initial treatment than steroids and is associated with less morbidity than curettage. We contend SDB could be considered a first-line therapy for UBCs in the humerus or femur in patients younger than 20 years requiring treatment for symptomatic UBCs. Patients with femoral cysts may require internal fixation to prevent fracture, and in those patients, curettage may be preferable, although we did not observe a benefit compared with SDB. Although our study was retrospective and contained selection bias, our subgroup analysis showed a trend in which, in both locations and across age groups, patients treated with steroids had higher failure rates than those treated by curettage or SDB. We observed curettage had a better chance of success than either injection as a second treatment; however, this difference was not significant and should be interpreted cautiously, especially given the potential morbidity of a more invasive procedure. Large, randomized clinical trials are needed to objectively compare SDB and other treatments for UBCs.

Acknowledgments We thank Kevin A. Raskin, MD, and Henry J. Mankin, MD, for valuable help and assistance.

\section{References}

1. Ahn JI, Park JS. Pathological fractures secondary to unicameral bone cysts. Int Orthop. 1994;18:20-22.
2. Altman DG. Practical Statistics for Medical Research. London, UK: Chapman and Hall; 1991:371-384.

3. Bumci I, Vlahovic T. Significance of opening the medullar canal in surgical treatment of simple bone cyst. J Pediatr Orthop. 2002;22:125-129.

4. Campanacci M, Capanna R, Picci P. Unicameral and aneurysmal bone cysts. Clin Orthop Relat Res. 1986;204:25-36.

5. Chang C, Stanton RP, Glutting J. Unicameral bone cysts treated by injection of bone marrow or methylprednisolone. J Bone Joint Surg Br. 2002;84:407-412.

6. Chigira M, Maehara S, Arita S, Udagawa E. The aetiology and treatment of simple bone cysts. J Bone Joint Surg Br. 1983;65:633-637.

7. Chigira M, Shimizu T, Arita S, Watanabe H, Heshiki A. Radiological evidence of healing of a simple bone cyst after hole drilling. Arch Orthop Trauma Surg. 1986;105:150-153.

8. Cohen J. Simple bone cysts: studies of cyst fluid in six cases with a theory of pathogenesis. J Bone Joint Surg Am. 1960;42:609616.

9. Cohen J. Intramedullary nailing for the treatment of unicameral bone cysts. J Bone Joint Surg Am. 2001;83:1279-1280.

10. de Palma L, Santucci A. Treatment of bone cysts with methylprednisolone acetate: a 9 to 11 year follow-up. Int Orthop. 1987;11:23-28.

11. de Sanctis N, Andreacchio A. Elastic stable intramedullary nailing is the best treatment of unicameral bone cysts of the long bones in children?: prospective long-term follow-up study. $J$ Pediatr Orthop. 2006;26:520-525.

12. Fahey JJ, O'Brien ET. Subtotal resection and grafting in selected cases of solitary unicameral bone cyst. J Bone Joint Surg Am. 1973;55:59-68.

13. Farber JM, Stanton RP. Treatment options in unicameral bone cysts. Orthopedics. 1990;13:25-32.

14. Garceau GJ, Gregory CF. Solitary unicameral bone cyst. J Bone Joint Surg Am. 1954;36:267-280.

15. Gebhart M, Blaimont P. Contribution to the vascular origin of the unicameral bone cyst. Acta Orthop Belg. 1996;62:137-143.

16. Harris EK, Albert A. Survivorship Analysis for Clinical Studies. New York, NY: Marcel Dekker; 1991:29-49.

17. Hosmer DW, Lemeshow S. Applied Logistic Regression. 2nd ed. New York, NY: John Wiley and Sons; 2000:11-64.

18. Jaffe HL, Lichtenstein L. Solitary unicameral bone cyst: with emphasis on the roentgen picture, the pathologic appearance and the diagnosis. Arch Surg. 1942;4:1004-1025.

19. Kanellopoulos AD, Yiannakopoulos CK, Soucacos PN. Percutaneous reaming of simple bone cysts in children followed by injection of demineralized bone matrix and autologous bone marrow. J Pediatr Orthop. 2005;25:671-675.

20. Katz MH. Multivariable Analysis: A Practical Guide for Clinicians. 2nd ed. New York, NY: Cambridge University Press; 2006:96-136.

21. Killian JT, Wilkinson L, White S, Brassard M. Treatment of unicameral bone cyst with demineralized bone matrix. J Pediatr Orthop. 1998;18:621-624.

22. Komiya S, Minamitani K, Sasaguri Y, Hashimoto S, Morimatsu M, Inoue A. Simple bone cyst: treatment by trepanation and studies on bone resorptive factors in cyst fluid with a theory of its pathogenesis. Clin Orthop Relat Res. 1993;287:204-211.

23. Lokiec F, Ezra E, Khermosh O, Wientroub S. Simple bone cysts treated by percutaneous autologous marrow grafting: a preliminary report. J Bone Joint Surg Br. 1996;78:934-937.

24. Mackley JT, Joyce MJ. Unicameral bone cyst (simple bone cyst). Orthop Clin North Am. 1989;20(3):407-415.

25. McKay DW, Nason SS. Treatment of unicameral bone cysts by subtotal resection without grafts. J Bone Joint Surg Am. 1977;59:515-519. 
26. Mirra JM, Bernard GW, Bullough PG, Johnston W, Mink G. Cementum-like bone production in solitary bone cysts (so-called "cementoma" of long bones): report of three cases. Electron microscopic observations supporting a synovial origin to the simple bone cyst. Clin Orthop Relat Res. 1978;135:295-307.

27. Neer CS, Francis KC, Johnston AD, Kiernan HA Jr. Current concepts on the treatment of solitary unicameral bone cyst. Clin Orthop Relat Res. 1973;97:40-51.

28. Neer CS 2nd, Francis KC, Marcove RC, Terz J, Carbonara PN. Treatment of unicameral bone cyst: a follow-up study of one hundred seventy-five cases. J Bone Joint Surg Am. 1966;48:731-745.

29. Oppenheim WL, Galleno H. Operative treatment versus steroid injection in the management of unicameral bone cysts. J Pediatr Orthop. 1984;4:1-7.

30. Peduzzi P, Concato J, Kemper E, Holford TR, Feinstein AR. A simulation study of the number of events per variable in logistic regression analysis. J Clin Epidemiol. 1996;49:1373-1379.

31. Roposch A, Saraph V, Linhart WE. Flexible intramedullary nailing for the treatment of unicameral bone cysts in long bones. J Bone Joint Surg Am. 2000;82:1447-1453.

32. Rougraff BT, Kling TJ. Treatment of active unicameral bone cysts with percutaneous injection of demineralized bone matrix and autogenous bone marrow. $J$ Bone Joint Surg Am. 2002;84:921-929.

33. Santori F, Ghera S, Castelli V. Treatment of solitary bone cysts with intramedullary nailing. Orthopedics. 1988;11:873-878.

34. Scaglietti O, Marchetti PG, Bartolozzi P. Final results obtained in the treatment of bone cysts with methylprednisolone acetate (depo-medrol) and a discussion of results achieved in other bone lesions. Clin Orthop Relat Res. 1982;165:33-42.

35. Shindell R, Huurman WW, Lippiello L, Connolly JF. Prostaglandin levels in unicameral bone cysts treated by intralesional steroid injection. J Pediatr Orthop. 1989;9:516-519.

36. Shinozaki T, Arita S, Watanabe H, Chigira M. Simple bone cysts treated by multiple drill-holes: 23 cysts followed 2-10 years. Acta Orthop Scand. 1996;67:288-290.

37. Snyder BD, Hauser-Kara DA, Hipp JA, Zurakowski D, Hecht AC, Gebhardt MC. Predicting fracture through benign skeletal lesions with quantitative computed tomography. J Bone Joint Surg Am. 2006;88:55-70.

38. Spence KF, Sell KW, Brown RH. Solitary bone cyst: treatment with freeze-dried cancellous bone allograft: a study of one hundred seventy-seven cases. J Bone Joint Surg Am. 1969;51: 87-96.

39. Spence KF Jr, Bright RW, Fitzgerald SP, Sell KW. Solitary unicameral bone cysts: treatment with freeze-dried crushed cortical-bone allograft: a review of one hundred and forty-four cases. J Bone Joint Surg Am. 1976;58:636-641.

40. Virchow R. Uber die Bildung von Knochencysten. Monatsberichte der Koniglich Preussischen Akademie der Wissenschafen. 1876;Suppl:369-381.

41. Wilkins RM. Unicameral bone cysts. J Am Acad Orthop Surg. 2000;8:217-224.

42. Yandow SM, Lundeen GA, Scott SM, Coffin C. Autogenic bone marrow injections as a treatment for simple bone cyst. J Pediatr Orthop. 1998;18:616-620. 